\title{
The Correlation between the Presence of Dehiscence or Fenestration and the Severity of Tooth Attrition in Contemporary Dry Japanese Adult Skulls-Part I
}

\author{
by \\ Toshimitsu EZAWA, Hiroshi SANO, Kazuo KANEKO, \\ Shigenori HIRUMA, Kenji FUJIKAWA and Seidai MURAI
}

(Received 28 November 1986)

\section{Introduction}

Dehiscence and fenestration (Fig. 1) have been discussed in a number of previous studies $^{[1-15]}$. Such defects of the thin alveolar bone have been suggested to be due to anatomical factors ${ }^{[6-8,11,12,15,16]}$ (buccolingual tooth malposition), occlusal function $^{[4,5]}$ or a number of other unidentified abnormal causes, but none of these have been clearly identified.

The purpose of the present study was to evaluate the frequency of dehiscence or fenestration, and to discuss its correlation with tooth attrition (occlusal factor) in contemporary dry Japanese skulls.

\section{Materials and Methods}

Both the upper and lower jaws of 188 dry Japanese skulls, the ages and sexes of which had been determined, were obtained from University Museum, Tokyo University and the Department of Anatomy of the Jikei University School of Medicine. Skulls which had artificial osseous destruction or osseous defects due to periodontitis were excluded from this study. A final total of 96 jaws of 24 male and 24 female skulls from subjects between 14 and 47 years of age were selected for study. The mean distance from the cementoenamel junction (CEJ) to the alveolar bone crest was within $2 \mathrm{~mm}$ in each skull (Fig. 2).

1) Measurement of bone level

The alveolar bone level was measured at the following reference points on each tooth $^{[17]}$. For incisors, canines or premolars, the level was measured at the middle points of both the facial and lingual surfaces. For molar teeth, the level was measured at four reference points which were located at $1 / 4$ of the cervical length from each of the mesial or distal line angles on both the facial and lingual surfaces (Fig. 3). Bone level was measured from the CEJ to the alveolar bone crest at each of the reference points using an originally designed metal ruler with a narrow tip graduated in units of $0.5 \mathrm{~mm}$. The average of these distances was calculated for each pair of jaws from each skull.

江澤敏光, 佐野裕士, 金子和夫, 蛭間重能, 藤川謙次, 村井正大：Department of Periodontics, Nihon University School of Dentistry. 


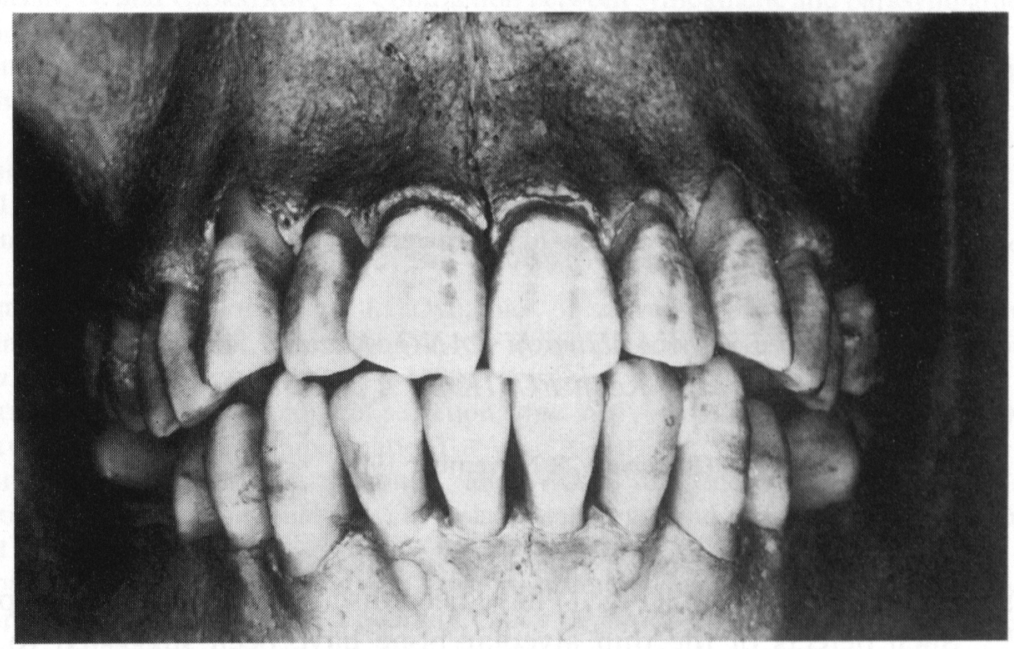

Fig. 1. The appearance of dehiscence and fenestration

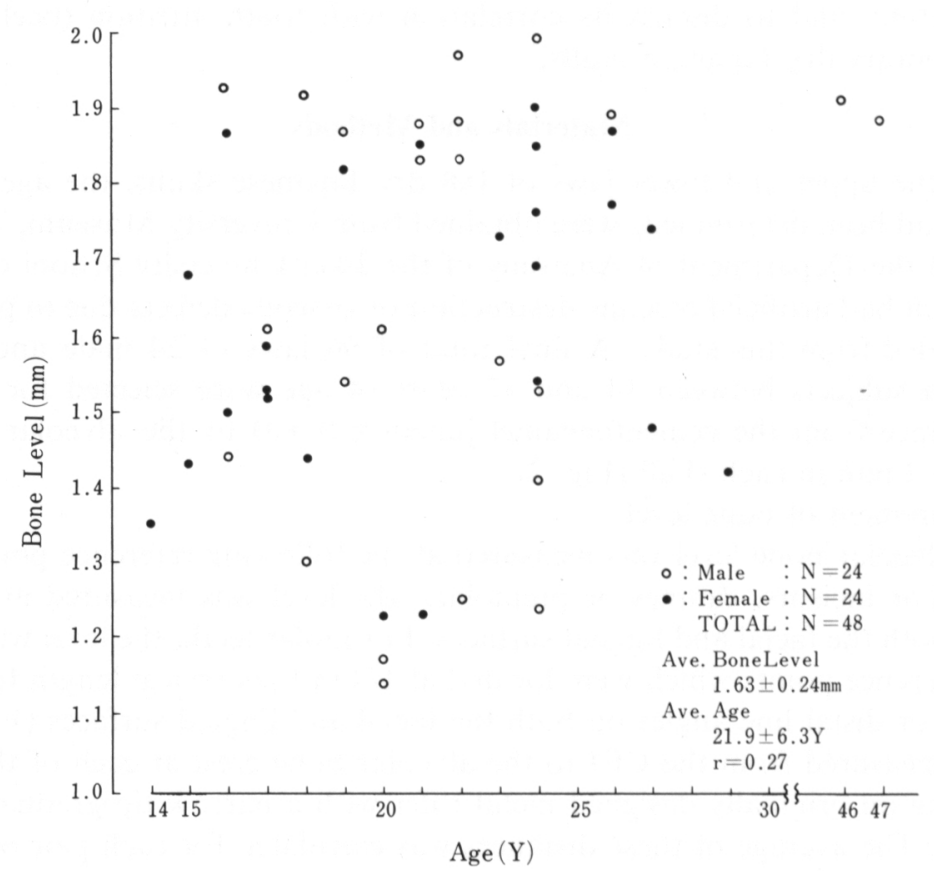

Fig. 2. Correlation between bone level and age 


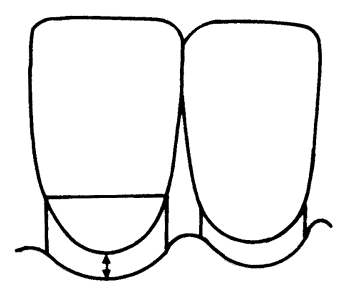

a: Reference point for incisor or premolar tooth

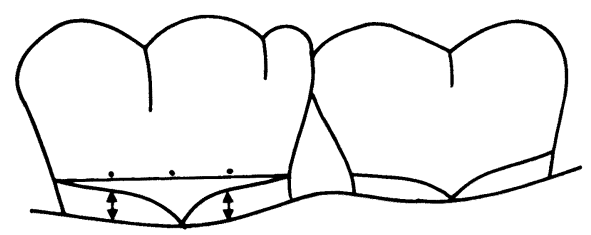

b: Reference point for molar tooth

Fig. 3

2) Criteria of tooth attrition ${ }^{[18]}$

The degree of tooth attrition for each type of tooth is described in Table 1.

3) Dehiscence and Fenestration

Dehiscence: A thin cleft-like alveolar bone located apically from an imaginary line keeping an appropriate distance from the CEJ. When the enamel projection is seen from some aspects, this imaginary line is estimated excluding this projection.

Fenestration: Fenestration is a circumscribed defect in the cortical plate, exposing a facial or lingual root surface. In addition, these defects and the surrounding areas do not show any thick alveolar bone.

The correlation of the alveolar bone level, tooth attrition, dehiscence and fenestration for each aspect of each skull were analyzed with a personal computer (NEC, PC-9801 M2).

\section{Results}

There was no correlation between age and bone level $(r=0.27)$ (Fig. 2). The correlation between the severity of tooth attrition and the presence of dehiscence and fenestration is shown in Table $2 a-2 c$.

Of a total of 1292 teeth from both males (629) and females (663), 1119 teeth $(86.6 \%)$ showed no dehiscence or fenestration. However, 173 teeth $(13.4 \%)$ exhibited either dehiscence $(5.1 \%)$ or fenestration $(8.0 \%)$, or both $(0.3 \%)$.

Of the 663 teeth of the male group, 37 teeth $(5.6 \%)$ showed dehiscence and 58 teeth $(8.7 \%)$ exhibited fenestration. Of the 629 female teeth, 29 teeth $(4.6 \%)$ showed dehiscence and 45 teeth $(7.2 \%)$ exhibited fenestration.

The Kolmogorov-Smirnov one-sample test ${ }^{[19]}$ was used to analyze the correlation between the severity of tooth attrition and the presence of dehiscence or 
Table 1 Degree of tooth attrition

Upper incisor

$\infty$ 1: isolated tooth attrition present

2: marginal ridge and incisal ridge worn out, linear dentin surface recognizable

or only enamel surface almost worn out

3: incisal ridge worn out and dentin surface clearly exposed

4: clear and widely exposed dentin surface apparent on the incisal ridge

Lower incisor

1: isolated tooth attrition present

eी 2: linear dentin surface exposed

$\infty$ 3: clear and widely exposed dentin surface apparent on the incisal ridge

Upper canine

e 4: exposed dentin surface also apparent on the mesial and distal marginal ridge

1: only cusp tip worn out

2: tooth attrition exposed on the mesial marginal ridge

3: tooth attrition generally recognizable on the incisal ridge and dentin surface slightly exposed

4: tooth attrition also recognizable on the lingual aspect and dentin surface widely exposed

Lower canine

1: only cusp tip worn out

Q 2: tooth attrition apparent on facial surface

3: tooth attrition apparent on incisal ridge

Upper premolar

4: dentin surface widely exposed

1: only cusp tip worn out

2: an isolated tooth attrition clealy recognizable

3: general tooth attrition apparent except for the mesial marginal ridge and

exposed dentin apparent on the facial cusp

4: general tooth attrition recognizable and dentin surface exposed at both the facial and lingual cusps

Lower premolar

1: only facial cusp worn out

(1) 2: tooth attrition recognizable along buccal ridge

$Q$ 3: most of the occlusal surface worn out and dentin surface exposed in isolated

areas

Upper molar

4: dentin surface widely exposed

1: isolated tooth attrition recognizable

: most of the occlusal surface worn out

: isolated dentin surface exposed

4: dentin surface widely exposed

Lower molar

1: isolated tooth attrition recognizable

2: most of the occlusal surface worn out

3: isolated dentin surface exposed

4: dentin surface widely exposed 
Table 2 Correlation between the severity of tooth attrition and the presence of dehiscence and fenestration

a: Total $(n=48)$

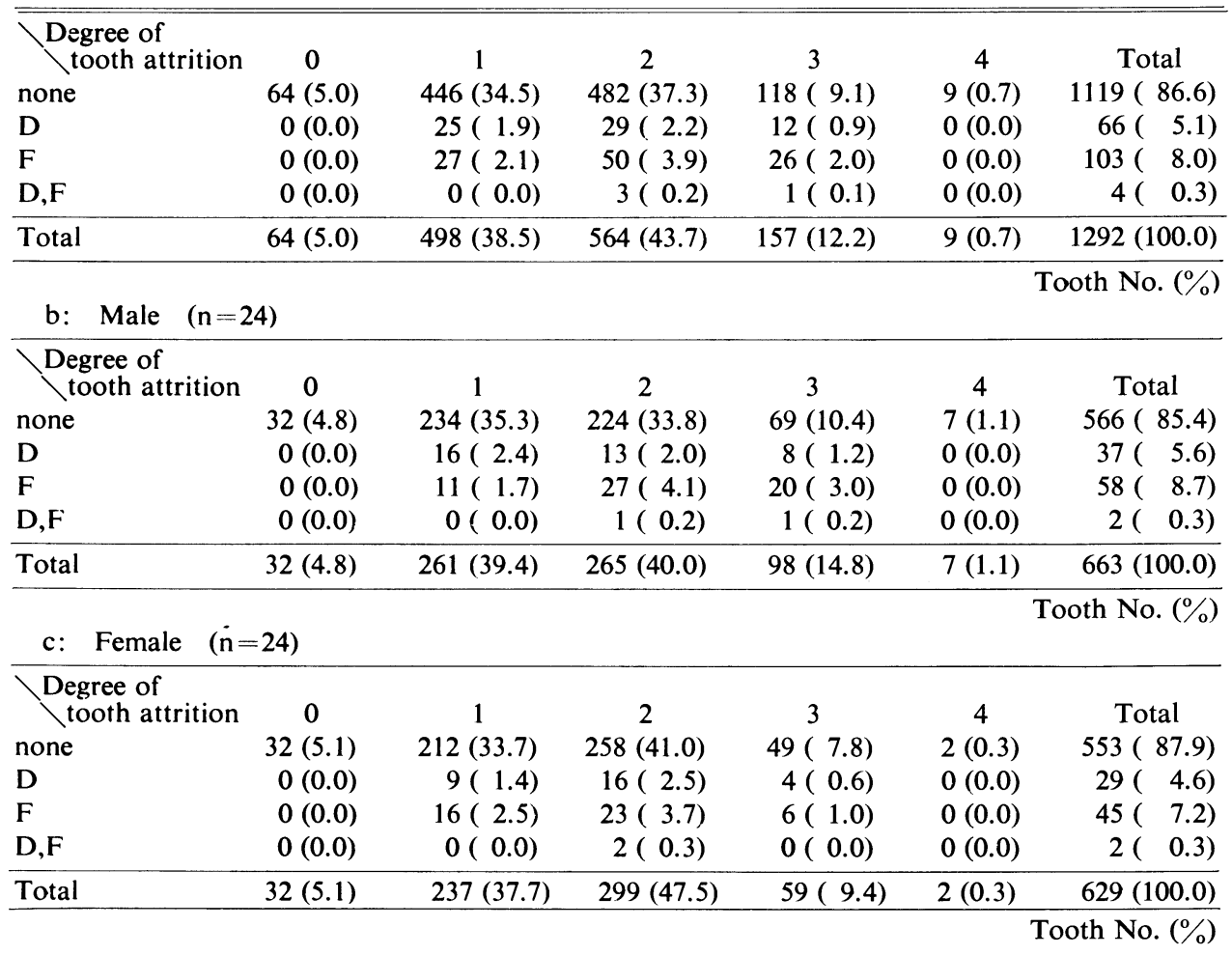

fenestration. No difference between the presence of dehiscence or fenestration and the severity of tooth attrition was adopted as a null hypothesis. After analyzing the correlation for each group, this hypothesis could not be abandoned for the presence of dehiscence in the male group and the presence of dehiscence and/or fenestration in the female group. However, this hypothesis was abandoned for the presence of fenestration in the male group. Therefore, the presence of dehiscence in the male group and the presence of dehiscence and/or fenestration in the female group showed no correlation with the severity of tooth attrition. In addition, the presence of fenestration in the male group did not show any clear correlation with the severity of tooth attrition.

\section{Discussion}

Dehiscence and fenestration in Japanese adult skulls have been described previously ${ }^{[1,2,7,13]}$. Dehiscence and fenestration in the upper jaw have frequently been recognized on canines, first premolars, first molars and second incisors. On the other hand, dehiscence has been seen on canines, first premolars or second incisors, while fenestration has been found for second incisors, canines, first premolars or central incisors in the lower jaw. The present study examined both dehiscence and 
fenestration from the facial or buccal aspects only, since these defects were only present to a slight degree on the lingual or palatal surface. At the healthy human dentogingival junction, the alveolar bone level has been recognized to be about one millimeter apical from the CEJ when the most coronal junctional epithelium is located on the $\mathrm{CEJ}^{[20]}$. On the other hand, previous studies have reported that in radiographs, an apical interproximal bone level of more than $2 \mathrm{~mm}$ can be classified as a pathological condition ${ }^{[21-25]}$. Generally, a bone level within $2.5 \mathrm{~mm}$ from the CEJ on a dry skull has been reported to represent a condition free of periodontal disease ${ }^{[26]}$.

The following factors possibly responsible for generating dehiscence or fenestration have been described in many studies; orthodontic forces ${ }^{[3]}$, tooth attri$\operatorname{tion}^{[4,5]}$, age ${ }^{[5,8,12]}$, thin alveolar bone $e^{[6,11]}$, inclination of the long axis of the tooth ${ }^{[7]}$, and tooth malposition ${ }^{[8,11]}$. However, orthodontic forces could be excluded from the present study, since the skulls had been obtained about 60 years previously.

There is no unified opinion regarding the presence of dehiscence or fenestration; the frequency of dehiscence or fenestration has been reported to be increased ${ }^{[5]}$ or decreased $^{[8,12]}$ in proportion with age. However, according to our previous research $^{[15]}$, the presence of dehiscence might have no correlation with the age of the specimen. Inclination of the long axis of the tooth and malposition of the tooth are closely related to the thickness of the alveolar bone. Therefore, thin alveolar bone and tooth attrition, which are related to occlusal force and trauma, might play a major role in generating dehiscence or fenestration.

According to our present study, 98 cases out of 633 in the male group showed grade 3 tooth attrition, and 20 teeth $(20.4 \%)$ exhibited the presence of fenestration. The Kolmogorov-Smirnov one-sample test showed a slight correlation between the severity of tooth attrition and the presence of dehiscence or fenestration. However, this relationship was not confirmed clearly for the following reasons: the low number of cases of grade 4 tooth attrition, and the low frequency of dehiscence or fenestration in other cases of grade 3 tooth attrition. Tooth attrition has been used for estimating age in the field of legal medicine because of the known correlation of the severity of tooth attrition with age ${ }^{[18]}$. From an epidemiologic viewpoint, the severity of alveolar bone loss increases with age ${ }^{[27,28]}$, although it is not correlated individually ${ }^{[16]}$.

KAKEHASHI et al. ${ }^{[4]}$ (1963) reported the effect of traumatic factors on the presence of dehiscence or fenestration following an examination of gorilla skulls. STAHL et al. ${ }^{[5]}$ emphasized the influence of occlusal trauma on the thickness of the alveolar bone which in turn was related to the presence of dehiscence or fenestration. However, KAKEHASHI et al. ${ }^{[4]}$ did not mention the criteria they used for dehiscence in their study. In addition, the alveolar bone level of Figure 5 in their paper ${ }^{[4]}$ looks like the result of a combination of dehiscence and osseous defects due to periodontitis. Therefore, we selected specimens that had a bone level within $2 \mathrm{~mm}$ in order to exclude cases of alveolar bone loss due to periodontitis and proposed criteria for the presence of dehiscence.

There has been shown to be no significant correlation between gingival recession and tooth mobility and between tooth mobility and dehiscence in humans ${ }^{[15]}$. 
In addition, there is no apparent correlation between the severity of tooth attrition and the presence of osseous defects ${ }^{[29,30]}$. Dehiscence or fenestration might be related to the thickness of the alveolar bone according to the results of the present study, except for the presence of fenestration in the male group. Future study of the correlation between tooth attrition and occlusal factors would probably be of more value, since tooth attrition of the first molars has been shown to be produced mainly during the stage of mixed dentition ${ }^{[31]}$.

\section{Conclusions}

After examination of 1292 teeth in 96 upper and lower jaws from contemporary dry Japanese skulls, the ages and sexes of which had been determined, the following results were obtained:

1. Of the 1292 teeth, $1119(86 \%)$ showed neither dehiscence nor fenestration, 66 $(5.1 \%)$ showed dehiscence, $103(8.0 \%)$ exhibited fenestration and $4(0.3 \%)$ showed both. In addition, 37 teeth $(5.6 \%)$ showed dehiscence and $58(8.7 \%)$ exhibited fenestration in the male group (663 teeth), while 29 teeth $(4.6 \%)$ showed dehiscence and $45(7.2 \%)$ exhibited fenestration in the female group (629 teeth).

2. There was no correlation between the presence of dehiscence or fenestration and the severity of tooth attrition except for fenestration in the male group which suggested a slight correlation.

\section{Acknowledgements}

The authors wish to acknowledge the advice and assistance of Prof. T. Kitagawa of the Department of Anatomy, Nihon University School of Dentistry, Prof. A. Yamauchi of the Department of Anatomy, University of Tokyo School of Medicine, and Associate Prof. S. Kato of the Department of Anatomy, the Jikei University School of Medicine, and the help provided by their departments.

\section{References}

[1] Kitamura, K.: Fenestration and dehiscence of the alveolar bone in dry Japanese skulls, Shikwa Gakuho, 46, 837-850, 1941 (in Japanese)

[2] SARAI, G.: Anatomical study of fenestration in the upper and lower jaw of dry Japanese skulls, Reports from the Department of Anatomy, Tokyo Jikeikai School of Medicine, 10, 1-14, 1953 (in Japanese)

[3] Nabers, C. L., Spear, G. R. and Beckham, L. C.: Alveolar dehiscence, Texas Dent. J., 78, 4-6, 1960

[4] Kakehashi, S., Baer, P. N. and White, C. L.: Comarpative pathology of periodontal disease, I. Gorilla, Oral Surg., 16, 397-406, 1963

[5] Stahl, S. S., Cantor, M. and Zwig, E.: Fenestration of the labial alveolar plate in human skulls, Periodontics, 1, 99-102, 1963

[6] Elliott, J. R. and Bowers, G. M.: Alveolar dehiscence and fenestration, Periodontics, 1, 245-248, 1963

[7] Tadakuma, T.: Anatomical study of Japanese adult skull 2. Socket, Shikwa Gakuho, 69, 665698, 1969 (in Japanese)

[8] LaRato, D. C.: Alveolar plate fenestration and dehiscences of the human skull, Oral Surg., 29, 816-819, 1970

[9] Larato, D. C.: Periodontal bone defect in the juvenile skull, J. Periodontol., 41, 473-475, 1970 
[10] Larato, D. C.: Alveolar plate defects in children's skulls, J. Periodontol., 43, 502, 1972

[11] Abdelmalek, R. G. and Bissada, N. F.: Incidence and distribution of alveolar bony dehiscence and fenestration in dry human Egyptian jaws, J. Periodontol., 44, 586-588, 1973

[12] Davies, R. M., Downer, M. C., Hull, P. S. and Lennon, M. A.: Alveolar defects in human skulls, J. Clin. Periodontol., 1, 107-111, 1974

[13] Kawasaki, K., Kobayashi, N., Tsukada, M., Hasegawa, M. and Hara, K.: Prevalence, location and extent of alveolar bony fenestration and dehiscence in dry human skulls, J. Japan. Assoc. Periodontol., 19, 31-40, 1977 (in Japanese)

[14] Edel, A.: Alveolar bone fenestrations and dehiscences in dry Bedouin jaws, J. Clin. Periodontol., 8, 491-499, 1981

[15] Bernimoulin, J.P. and Curilovic, Z.: Gingival recession and tooth mobility, J. Clin. Periodontol., 4, 107-114, 1977

[16] Ezawa, T., Sano, H., Okuno, K., Furukawa, K., Ito, K. and Murai, S.: Correlation between bone level and age in contemporary dry Japanese skulls, J. Japan. Assoc. Periodontol., 27, 610-617, 1985 (in Japanese)

[17] EzawA, T.: The thickness and form of alveolar bone in contemporary dry Japanese skulls, J. Japan. Assoc. Periodontol., 26, 243-256, 1984 (in Japanese)

[18] TAKeI, T.: The use of tooth attrition in age estimation, Jpn. J. Legal. Med., 24, 4-17, 1970 (in Japanese)

[19] Siegel, S.: Nonparametric statistics: For the behavioural sciences, New York: McGrawHill Book Co. Ltd., 1956

[20] Gargiulo, A. W., Wentz, F. M. and Orban, B.: Dimensions and relations of the dentogingival junction in humans, J. Periodontol., 32, 261-267, 1961

[21] JoRkJend, L. and Birkeland, J. M.: Alveolar bone loss in the permanent first molars of Norwegian schoolchildren receiving systematic dental care, Community Dent. Oral Epidemiol., 4, 22-24, 1976

[22] Hakkarainen, K. and Ainamo, J.: Influence of overhanging posterior tooth restorations on alveolar bone height in adults, J. Clin. Periodontol., 7, 114-120, 1980

[23] Gjermo, P., Bellini, H. T., Santos, V. P., Martins, J. G. and Ferracyoli, J. R.: Prevalance of bone loss in a group of Brazilian teenagers assessed on bite-wing radiographs, J. Clin. Periodontol., 11, 104-113, 1984

[24] Hansen, B. F., Gjermo, P. and Bergwitz-Larsen, K. R.: Periodontal bone loss in 15-yearold Norwegians, J. Clin. Periodontol., 11, 125-131, 1984

[25] Tanaka, K., Tanaka, K., Tsujl, Y., Yoshinuma, N., Ito, K. and Murai, S.: Radiographic study of vertical bone loss due to interproximal open contacts and tooth mobility in periodontal disease, J. Japan. Assoc. Periodontol., 27, 168-178, 1985 (in Japanese)

[26] Davies, D. M., Picton, D. C. A. and Alexander, A. G.: An objective method of assessing the periodontal condition in human skulls, J. Periodont. Res., 4, 74-77, 1969

[27] Greene, J. C.: Oral hygiene and periodontal disease, Am. J. Pub. Health, 53, 913-922, 1963

[28] Scherp, H. W.: Current concepts in periodontal disease research: Epidemiological contributions, J. Am. Dent. Assoc., 68, 667-675, 1964

[29] Baer, P. N., Kakehashi, S., Littleton, N. W., White, C. L. and Lieberman, J. E.: Alveolar bone loss and occlusal wear, J. Am. Soc. Periodontol., 1, 91-98, 1963

[30] Abe, Y., Hiruma, S., Michiue, Y., Shinada, M. and Nakamura, J.: Study of attrition and alveolar bone loss, Jap. J. Conserv. Dent., 20, 187-194, 1976 (in Japanese)

[31] Ingervall, B.: Tooth contacts on the functional and nonfunctional side in children and young adults, Archs. Oral Biol., 17, 191-200, 1972 